\title{
SCTR regulates cell cycle-related genes toward anti-proliferation in normal breast cells while having pro-proliferation activity in breast cancer cells
}

\author{
SEONGEUN KANG ${ }^{1,3}$, BYUNGTAK KIM ${ }^{1}$, HAN-SUNG KANG ${ }^{2}$, GOOKJOO JEONG ${ }^{1}$, \\ HANSOL BAE ${ }^{1}$, HYUNKYUNG LEE ${ }^{1}$, SEUNGYEON LEE ${ }^{1}$ and SUN JUNG KIM ${ }^{1}$ \\ ${ }^{1}$ Department of Life Science, Dongguk University-Seoul, Goyang; \\ ${ }^{2}$ Research Institute and Hospital, National Cancer Center, Goyang, Republic of Korea
}

Received July 11, 2015; Accepted August 26, 2015

DOI: 10.3892/ijo.2015.3164

\begin{abstract}
Secretin receptor (SCTR), the G-protein coupled receptor (GPCR) for secretin, has been observed to be upregulated in a few tumor types while downregulated in others, promoting or suppressing the proliferation of tumor cells, respectively. However, little is known about the molecular regulatory mechanism of dysregulation in cancer. In the present study, an analysis of the biological pathways affected by methylation in breast cancer using the methylome databases revealed that GPCRs played a major part in the affected pathway. SCTR, one of the dysregulated GPCRs, showed hypermethylation $(\mathrm{P}<0.01)$ and downregulation $(\mathrm{P}<0.05)$ in breast cancer tissues. Pathway analysis after the downregulation of SCTR by siRNA in MCF-10A cells identified the G2/M stage checkpoint as the top-scored pathway. Cell cycle-related genes were all upregulated or downregulated suppressing cell proliferation. However, the overexpression of SCTR in MCF-7 cells led to a $35 \%$ increase of the cell proliferation index and 2.1-fold increase of cellular migration. Our findings indicate that SCTR suppresses the proliferation of normal breast cells, while the gene stimulates the proliferation and migration of cancer cells being downregulated by promoter methylation.
\end{abstract}

Correspondence to: Professor Sun Jung Kim, Department of Life Science, Dongguk University-Seoul, Goyang 410-820, Republic of Korea

E-mail: sunjungk@dongguk.edu

Present address: ${ }^{3}$ Aprogen Inc., Seongnam 462-807, Republic of Korea

Abbreviations: SCTR, secretin receptor; $\mathrm{CpG}$, cytosine guanine dinucleotide; GPCR, G-protein coupled receptor; MSP, methylationspecific PCR; RT-PCR, reverse transcription-polymerase chain reaction

Key words: breast cancer, cell cycle, $\mathrm{CpG}$ methylation, G-protein coupled receptor, network analysis, secretin receptor

\section{Introduction}

G-protein coupled receptors (GPCRs) are membrane-spanning protein receptors characterized by a variable number of ligands that include endogenous neurotransmitters and hormones, but also exogenous natural and artificial compounds $(1,2)$. More than 600 GPCRs have been identified in humans, and their activation results in diverse physiological changes. Upon binding to receptors, agonists trigger the activation of signaling pathways; whereas, antagonists impede the agonistmediated activation of the receptors. Inverse agonists, besides interfering with agonists as do antagonists, suppress the constitutive activity of receptors $(3,4)$.

In many cases, the dysregulation of GPCRs has been linked to the development of cancer, and hence, these proteins are of great interest to academia and the pharmaceutical industry. The overexpression of GPCRs has been revealed in a variety of cancer types. For example, an elevated GPER protein expression was revealed in embryonal carcinomas as well as in testicular stromal neoplasms $(5,6)$. CXCR4, one of the best established chemokine receptors, is highly expressed in breast cancer cells (7). The HER2/Neu oncogene, which occurs in $\sim 30 \%$ of breast cancers, limits the degradation of CXCR4, leading to its increased expression. In certain malignancies, some GPCRs and G proteins may play tumorsuppressive roles, and mutations may reflect the inactivation of the respective genes. For example, inactivating mutations in the melanocortin 1 receptor (MC1R), which is important for pigment production, increase the risk of melanoma development (8). Additionally, SIP2 receptor signaling through $\mathrm{G \alpha}_{13}$ in diffuse large B cell lymphoma (DLBCL) may exert tumorsuppressive functions (9).

Secretin receptor (SCTR) is a GPCR to which secretin binds, and it has long been known to mediate the effect of the gastrointestinal hormone on digestion and water homeostasis $(10,11)$. In physiological conditions, SCTR is highly expressed in secretin target organs (e.g., pancreas, kidney and small intestine), and it is expressed in the distal lung regions and liver, with trace levels in the brain, heart and ovaries (12). The dysregulation of SCTR and consequent diseases have been documented in limited cases. High SCTR expression has 
been observed in pancreatic ductal adenocarcinomas, cholangiocellular carcinomas, gastrinomas and bronchopulmonary carcinoid tumors (13-15). In contrast, SCTR has been found to be downregulated in colorectal cancer (16) and prostate cancer (17).

Many tumor-related genes are known to be regulated by epigenetic mechanisms, including DNA methylation. Genes that are downregulated and hypermethylated in breast cancer include BRCA1, PTEN and RASSF1 (18-20). Regarding SCTR, its CpG island methylation has not yet been extensively reported in cancer. Only a large chromosomal region at 2 q14.2 that spans EN1, SCTR and INHBB is known to be silenced in colorectal cancer (16).

In the present study, using an in silico approach, we found that SCTR was downregulated in all stages of breast cancer cell lines. After confirming the downregulation in cancer tissue, we tried to elucidate the epigenetic mechanism of the dysregulation by examining the promoter methylation. Furthermore, the gene was induced to be downregulated using siRNA, and a gene interaction network was constructed from the affected gene pool. Finally, the cell proliferation and migration activities of SCTR were monitored based on the information from the siRNA-knockdown experiment.

\section{Materials and methods}

In silico database analysis. The Infinium Methylation Chip data for a normal breast cell line, MCF-10A, and 10 cancer cell lines ranging from tumor stage I-IV were obtained from the GEO database (http://www.ncbi.nlm.nih.gov/geo/). The adopted accession number for normal breast cell is GSM443815. In cancer cells, the data used in stage I is GSM443818, stage II is GSM443811, GSM443817, GSM443812, GSM443819 and GSM664892, stage III is GSM443813 and GSM443814 and stage IV is GSM443816, GSM664906 and GSM443821. The Illumina Infinium Methylation Assay detects genome-wide methylation covering 27,578 CpG sites in 14,495 genes. Observations with adjusted P-values $\geq 0.05$ were removed and thus excluded from further analysis. Following adjustment, the genes of the cancer cell lines were defined as differentially methylated if they displayed an increased or decreased methylation level of at least 2-fold compared to that of the normal cell line. The Student's t-test was used to detect differences in mean levels of methylation and the expression level between the normal and cancer tissues using SPSS for Windows, version 17.0 (SPSS Inc., Chicago, IL, USA). P-values <0.05 were considered statistically significant.

Cell culture and transfection. The breast cancer cell line MCF-7 and the normal breast cell line MCF-10A, were purchased from the American Type Culture Collection (ATCC; Manassas, VA, USA). MCF-10A was grown in MEBM (Lonza, Basel, Switzerland) supplemented with MEGM SingleQuots (Lonza) and cholera toxin (List Biological Laboratories, Inc., Campbell, CA, USA). MCF-7 was grown in Dulbecco's modified Eagle's medium supplemented with $10 \%$ fetal bovine serum (FBS). All cells were cultured on the surface of a $75-\mathrm{cm}^{2}$ culture flask.

siRNA-based downregulation of SCTR was carried out by transiently transfecting the siRNA against SCTR into the MCF-10A cell cultures in a $60-\mathrm{mm}$ culture dish. siRNA of the gene and a control siRNA were purchased from SigmaAldrich (St. Louis, MO, USA) and added to the culture media up to $200 \mathrm{nM}$ in final concentration using a Lipofectamine RNAiMAX reagent (Invitrogen, Carlsbad, CA, USA). The cells were harvested $48 \mathrm{~h}$ after transfection for RNA isolation.

To establish stable cell lines expressing SCTR, $2 \mu \mathrm{g}$ of SCTR-expressing vector (GeneCopoeia, Germantown, MA, USA) was transfected into $1 \times 10^{6}$ of MCF-7 cells in a $75-\mathrm{cm}^{2}$ culture flask using Lipofectamine 2000 (Invitrogen) according to the manufacturer's instructions. A vector without the gene was used as a control. Two days after transfection, neomycin (400 $\mu \mathrm{g} / \mathrm{ml})$ (Gibco-BRL, Carlsbad, CA, USA) was added to select stable transfectants. The expression of SCTR was monitored by reverse transcription-polymerase chain reaction (RT-PCR).

Study subjects. Twenty-one pairs of breast cancer ( $\mathrm{BrCa})$ specimens and their corresponding adjacent normal tissue specimens were obtained from patients who had undergone surgery between 2011 and 2012 at the National Cancer Center (NCC) in Korea. All patients provided written informed consent to donate removed tissue to the NCC, and samples were obtained according to protocols approved by the Research Ethics Board of the NCC. BrCa specimens were subjected to histological examination by an expert pathologist for independent confirmation of tumor grade.

Real-time RT-PCR. Total RNA from $\sim 100 \mathrm{mg}$ of tissue was isolated using TRIzol reagent according to the manufacturer's protocol (Gibco-BRL) with a final suspension in $30 \mu \mathrm{l}$ of distilled water. Total RNA from a $75-\mathrm{cm}^{2}$ culture flask was isolated using the RNeasy Plus Mini kit (Qiagen) with a final elution in $30 \mu \mathrm{l}$ of distilled water. Reverse transcription was conducted using 1-5 $\mu \mathrm{g}$ of total RNA with a reverse transcription kit (Toyobo, Osaka, Japan). Gene expression was measured by real-time quantitative RT-PCR analysis using a Kapa SYBR FAST qPCR kit (Kapa Biosystems, Inc., Woburn, MA, USA) on an ABI 7300 instrument (Applied Biosystems, Foster City, CA, USA). One microliter of cDNA was used for PCR, which was performed in duplicate. The primers used for SCTR are forward, 5'-GATGTCACCTACTGCGATGC-3' and reverse, 5'-ACAAAAATGGCTGGAGAACC-3'. RNA quantity was normalized to GAPDH content, and gene expression was quantified according to the $2^{-\Delta \mathrm{Ct}}$ method. Primer sequences for GAPDH are forward, 5'-CAGGAGGCATTGCTGATGAT-3' and reverse, 5'-GAAGGCTGGGGCTCATTT-3'.

Methylation-specific PCR. Chromosomal DNA from $100 \mathrm{mg}$ of tissue samples was isolated using a genomic DNA purification kit (Promega, Madison, WI, USA) according to the manufacturer's protocol with a $60-\mu 1$ elution volume. Sodium bisulfite modification of genomic DNA and PCR were carried out as previously described (18). Briefly, $0.1 \mathrm{mg}$ of DNA was treated with sodium bisulfite, and then PCR was carried out using primers and a Kapa SYBR FAST qPCR kit (Kapa Biosystems). The primer sequences of methylated SCTR are forward, 5'-TTTGGAGTTTACGGATAGAAAGC-3' and reverse, 5'-CCGAAAATAAATATTATCAAACGTA-3'. Unmethylated SCTR are forward, 5'-TTTGGAGTTTATGG ATAGAAAGTGT-3' and reverse, 5'-TCCAAAAATAAAT 
ATTATCAAACATA-3'. A methylation index was calculated for each sample using the following formula: Methylation index $=1 /\left[1+2^{- \text {(CTu-CTme) }}\right] \times 100 \%$, where CTu and CTme are the cycle threshold obtained using the unmethylated and the methylated primer pair, respectively.

Expression microarrays and pathway analysis. An Illumina HumanHT-12 v4 Expression BeadChip (Illumina, San Diego, CA, USA) analysis covering 31,000 human genes and containing 47,231 probes was performed by Macrogen (Seoul, Korea). Briefly, biotinylated cRNA was prepared from $550 \mathrm{ng}$ of total RNA using the Illumina TotalPrep RNA amplification kit (Ambion, Austin, TX, USA). Fluorescent signals were obtained by scanning with iScan System, and data were extracted with Gene Expression Module 1.9.0 in GenomeStudio v2011.1 (Illumina). Data were processed by excluding genes with the detection P-values $\geq 0.05$, and differentially expressed genes displaying at least a 2-fold difference between the control cells and the siRNA treated cells were obtained. The array data were uploaded to the Gene Expression Omnibus (GEO) database, and they can be accessed via their website (http:// www.ncbi.nlm.nih.gov/geo/; accession number GSE60750).

Functional categorization and pathway construction for the gene pool obtained from the affected genes by siRNA or overexpressed SCTR were performed using the Ingenuity Pathway Analysis (IPA) software tool (Ingenuity Systems, Redwood City, CA, USA) (16). P-values for individual networks were obtained by comparing the likelihood of obtaining the same number of transcripts or greater in a random gene set as were actually present in the input file (i.e., the set of genes differentially methylated in normal and tumor tissue) using Fisher's exact test, based on the hypergeometric distribution. The highest confidence functional network was designated as the top network.

Cell proliferation and migration analysis. Cells cultured in a $60-\mathrm{mm}$ dish at $\sim 80 \%$ confluence were harvested using $0.05 \%$ Trypsin-EDTA (Gibco-BRL), washed with PBS, and then fixed in ice-cold $70 \%$ ethanol overnight. The cells were treated with $50 \mu \mathrm{g} / \mathrm{ml}$ RNaseA (Sigma-Aldrich) for $1 \mathrm{~h}$ and then stained with $50 \mu \mathrm{g} / \mathrm{ml}$ propidium iodide (Sigma-Aldrich) in the dark for $30 \mathrm{~min}$ at room temperature. Samples were analyzed using a FACSCanto II flow cytometer (Becton-Dickinson, Franklin Lakes, NJ, USA) reading with a 488-nm laser. The cell proliferation index was calculated using the following formula: Proliferation index $=(\mathrm{S}+\mathrm{G} 2+\mathrm{M}) /(\mathrm{G} 0 / \mathrm{G} 1+\mathrm{S}+\mathrm{G} 2+\mathrm{M}) \times 100 \%$, where each letter represents the number of cells at each stage.

For cell migration assay, cells were plated onto $8.0 \mu \mathrm{m}$ Transwell Inserts (Corning Life Sciences, Tewksbury, MA, USA) and cultured following the supplier's protocol. The migrated cells were stained with crystal violet and counted under a microscope. The migration ratio was calculated by dividing the number of migrated cells by the number of cells seeded.

\section{Results}

Various GPCRs are aberrantly methylated in breast cancer. To identify novel GPCRs that are deregulated by aberrant promoter methylation in breast cancer, a series of in silico anal-
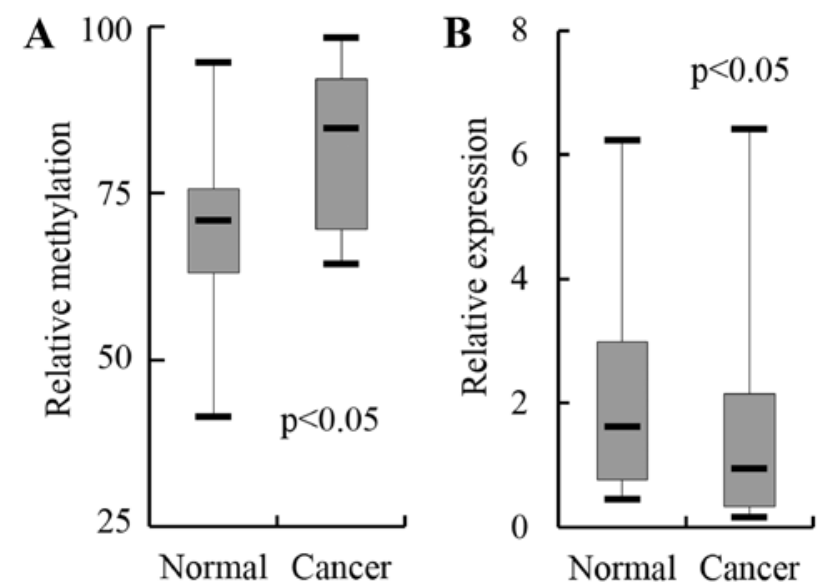

Figure 1. Methylation and expression analysis of SCTR in breast tumor tissues. Methylation levels of the $\mathrm{CpGs}$ at the promoter (A) and expression of SCTR (B) were examined by MSP and real-time RT-PCR for 21 pairs of tumor tissues and their surrounding normal tissues, respectively. The results are presented as box plots. Each sample was examined in duplicate, and the average relative methylation and expression levels were used for plotting.

ysis was performed on genome-wide methylation databases. The Illumina methylation assay data from a normal breast cell line and 10 breast cancer cell lines of stage I-IV were extracted from the GEO database. After filtrating out statistically insignificant $\mathrm{CpG}$ sites $(\mathrm{P}>0.05)$, the $\beta$-values of remaining $\mathrm{CpGs}$, indicative of the methylation level, were compared between the normal cell line and each cancer cell line. Screening of $\mathrm{CpG}$ sites fitting our criteria with 2-fold higher or lower methylation in cancer than in normal cells presented 808-1,517 CpGs which included 114 GPCRs. The GPCRs that showed the highest change of hypermethylation and hypomethylation were LPAR2 (33.3-fold) and GPR133 (-18.8-fold), GALR3 (19.9-fold) and GPR135 (-20.3-fold), QRFPR (20.9-fold) and CELSR1 (-21.3-fold), and GPR56 (16.7-fold) and BDKRB2 (18.1-fold) at stages I-IV, respectively.

SCTR is hypermethylated and downregulated in breast cancer. Among the aberrantly methylated GPCRs, the ones that frequently appear throughout the four cancer stages were selected for further examination. Twenty-four GPCRs (e.g., GPR135, TAS1R2 and SCTR) hit more than three stages (Table I). Three GPCRs, TAS1R2, F2RL2 and SCTR, whose methylation status is not yet known in any cancer tissue, were selected for further examination in breast cancer tissue. Twenty-one pairs of tumor and nearby normal tissue were examined by real-time methylation-specific PCR (MSP) and RT-PCR to monitor methylation and expression, respectively. The results indicated that only SCTR showed higher methylation levels $(\mathrm{P}<0.05)$ and downregulation $(\mathrm{P}<0.05)$ in cancer tissues than in normal tissues with a statistical significance (Fig. 1). SCTR, therefore, was selected to further address its role in breast cancer.

SCTR regulates cell cycle-related genes. To address the role of SCTR in breast cancer, knockdown was induced by transiently transfecting siRNA, which targeted the message in the MCF-10A cells, and then genome-wide expression change was monitored through microarray analysis. In total, 1,587 genes 
Table I. GPCRs displaying differential methylation at breast tumor stage I-IV.

Fold change $\mathrm{e}^{\mathrm{a}}$

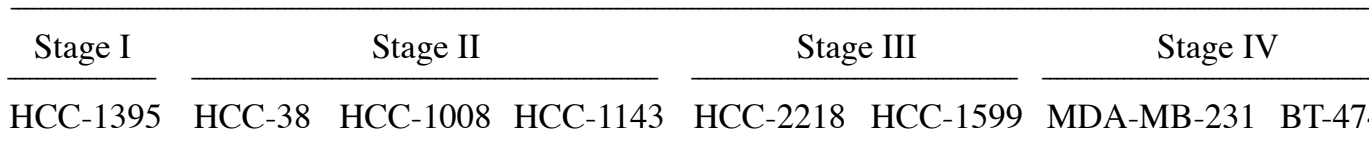

Gene Accession no. HCC-1395 HCC-38 HCC-1008 HCC-1143 HCC-2218 HCC-1599 MDA-MB-231 BT-474

\begin{tabular}{|c|c|c|c|c|c|c|c|c|c|}
\hline GALR3 & NM_003614.1 & 19.9 & 19.4 & 18.1 & 19.7 & 19.2 & 15.1 & 12.4 & 19.7 \\
\hline MC4R & NM_005912.1 & 17.9 & 19.7 & 1.3 & 16.2 & 19.8 & 19.5 & 16.0 & 19.0 \\
\hline QRFPR & NM_198179.1 & 13.2 & 20.9 & -1.1 & 11.5 & 20.3 & 22.6 & 22.5 & 22.2 \\
\hline GIPR & NM_000164.2 & 12.2 & 11.4 & 3.2 & 4.5 & 12.0 & 11.1 & 9.8 & 13.2 \\
\hline F2RL2 & NM_004101.2 & 9.8 & 11.5 & 7.5 & 10.2 & 7.9 & 10.9 & -1.0 & 10.7 \\
\hline FFAR2 & NM_005306.1 & 9.4 & 9.1 & -1.9 & 5.5 & 7.8 & -2.0 & 4.6 & -2.6 \\
\hline HTR2A & NM_000621.2 & 8.8 & 4.3 & 1.2 & 7.5 & 5.2 & 7.7 & 2.7 & 4.2 \\
\hline GHSR & NM_198407.1 & 6.4 & -3.1 & -1.2 & 6.3 & 6.3 & 6.3 & 5.7 & 6.3 \\
\hline TAS1R2 & NM_152232.1 & 6.2 & 6.2 & 4.8 & 4.6 & 6.0 & 6.1 & 3.9 & 6.3 \\
\hline CRHR2 & NM_001883.2 & 5.7 & 4.5 & -2.9 & 5.3 & 2.1 & 2.1 & 7.5 & 3.7 \\
\hline SCTR & NM_002980.1 & 5.4 & 5.8 & -2.6 & 5.5 & 5.5 & 5.7 & 1.3 & 5.7 \\
\hline GPR78 & NM_080819.2 & 1.4 & -3.1 & -3.2 & -4.8 & -1.1 & 2.1 & -1.4 & 2.1 \\
\hline GPR135 & NM_022571.4 & 1.0 & -20.3 & -10.0 & -1.1 & -17.3 & -18.9 & -13.5 & -18.7 \\
\hline GPR63 & NM_030784.1 & -1.1 & -18.5 & -21.1 & -1.5 & -8.1 & -1.9 & -1.1 & -1.1 \\
\hline CELSR1 & NM_014246.1 & -1.5 & -21.3 & -12.9 & -1.1 & -17.1 & -20.6 & -2.2 & -22.8 \\
\hline GPR160 & NM_014373.1 & -1.8 & -2.0 & -12.6 & -1.2 & -1.8 & -49.4 & -2.0 & -17.5 \\
\hline HTR1E & NM_000865.1 & -1.9 & -20.0 & -23.8 & -11.3 & -13.5 & -3.6 & -1.9 & -1.8 \\
\hline GALR1 & NT_025004.13 & -2.5 & -2.6 & -3.1 & -1.5 & -1.0 & 1.0 & 1.0 & -5.0 \\
\hline NTSR1 & NM_002531.1 & -4.7 & -18.8 & -18.9 & -2.6 & -1.2 & 1.0 & -1.0 & -2.8 \\
\hline FZD10 & NM_007197.2 & -6.6 & -10.4 & -51.8 & -9.0 & -1.2 & -4.7 & 1.0 & 1.0 \\
\hline RXFP3 & NM_016568.1 & -7.2 & -31.1 & -27.9 & -1.9 & -24.2 & -1.8 & -1.1 & -1.6 \\
\hline SSTR5 & NM_001053.1 & -7.4 & -7.7 & -56.2 & -3.8 & -2.1 & -1.8 & 1.0 & -17.6 \\
\hline GPR133 & NM_198827.2 & -18.8 & -1.5 & -74.1 & -1.0 & -10.9 & -1.1 & -3.8 & -1.0 \\
\hline GPR37 & NM_005302.2 & -23.2 & -57.7 & -48.1 & -80.1 & -64.8 & -44.9 & -9.6 & -87.1 \\
\hline
\end{tabular}

${ }^{\text {a}}$ Fold change is expressed as the ratio of methylation level between the indicated cancer cell line vs. the normal cell line, MCF-10A. Negative values denote for decreased methylation level in cancer cells.

fitting our criteria of expression change $>2$-fold with 993 upregulated and 594 downregulated genes were submitted to the IPA. The results indicated the 'cellular movement, cancer, connective tissue disorders' as the top network and 'cell cycle: G2/M DNA damage checkpoint regulation' as the top pathway, implying its role in tumorigenesis (Fig. 2 and Table II). Notably, the majority of the cell cycle-related genes in the network were upregulated or downregulated in a way increasing cell proliferation after SCTR was downregulated by siRNA, suggesting SCTR has anti-proliferative activity in normal cells. In the network, three kinases, TNK2 (ACK1), DDR1 and CDCP1, all of which are known to be oncogenic proteins, were upregulated (21-23). Whereas, DICER1, TPM1 and RGS2, all of which are known to be related with tumor-suppressing activity, were downregulated in the network (24-26). Therefore, it is expected that the genes would show upregulation or downregulation in cancer if they are oncogenic or tumor-suppressive, respectively. The in silico expression analysis based on the Oncomine data- base platform confirmed the upregulation of TNK2 (ACK1), DDR1, and CDCP1 and the downregulation of DICER1, TPM1 and RGS2 in the breast cancer cells (Fig. 3).

To examine the cell cycle-related activity of SCTR, the MCF-10A normal breast cells wherein the SCTR was downregulated by siRNA were monitored for cell proliferation index by FACS analysis. The result indicated, unexpectedly, that no remarkable change of the cell proliferation index was observed (Fig. 4). To determine whether the effect of SCTR on the cell cycle differs between normal and cancer cells, the gene was overexpressed in the MCF-7 cancer cells by stable transfection (Fig. 5A), and the cells were monitored for cellcycle status (Fig. 5B). The result indicated a 35\% increase of the cell proliferation index with a $9 \%$ decrease of G1 phase cells but a $39 \%$ increase of $\mathrm{S}$ and G2/M phase cells. Next, the effect of SCTR on the migration of the cancer cells was examined for the SCTR-overexpressing MCF-7 cells, and the cells showed a 2.1-fold increase of migration compared to the MCF-7 cells (Fig. 5C). These facts suggest that SCTR 


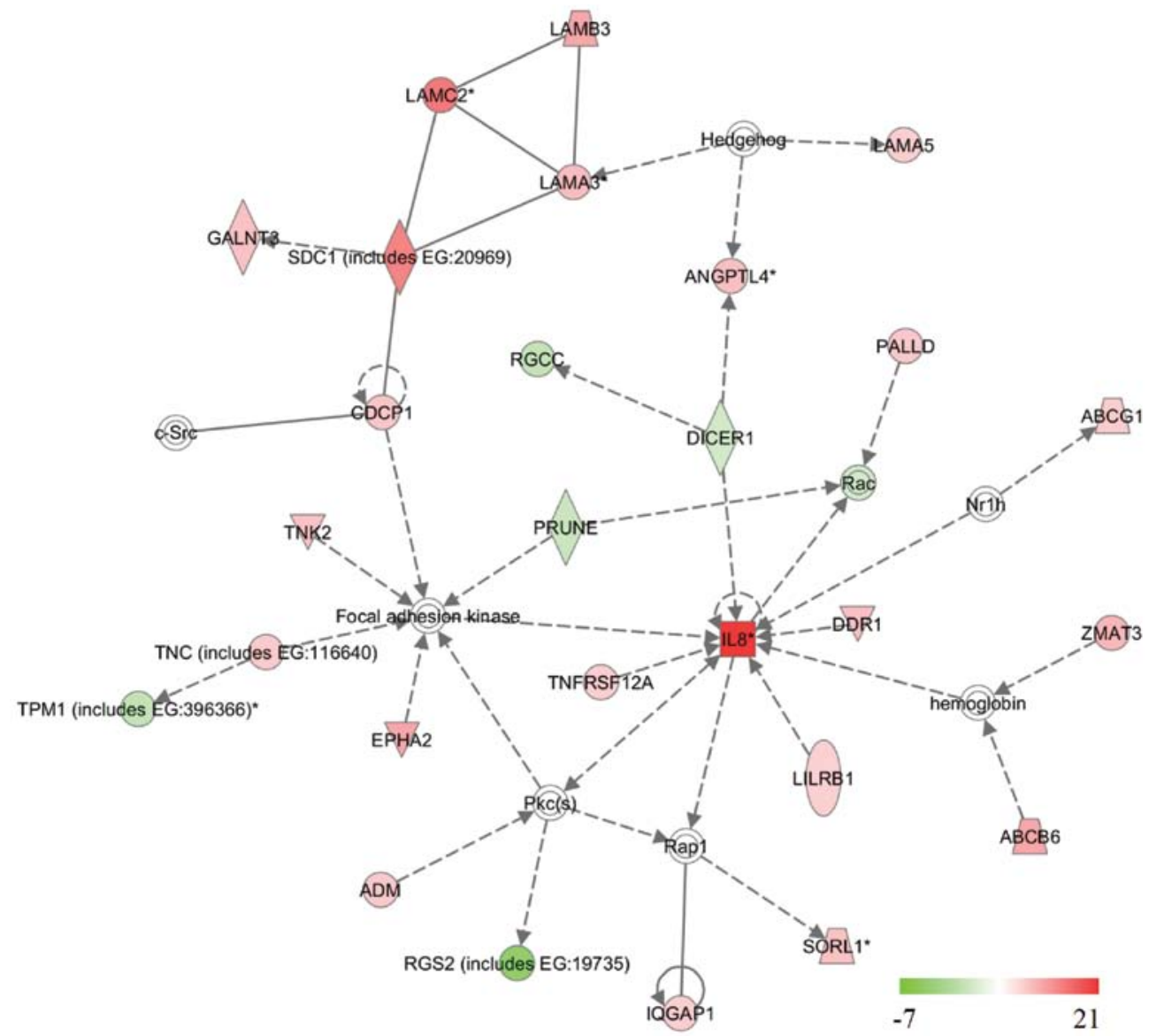

Figure 2. Genome-wide effect of downregulation of SCTR by siRNA. SCTR was knocked down in MCF-10A cells by siRNA, and the affected genes were identified by the IPA network after an expression array. The network is the highest confidence network of genes displaying altered expression levels by siRNA. In the network, upregulated genes are red, while downregulated genes are shown in green. The intensity of the color reflects the magnitude of expression change. According to IPA, the network is relevant to 'cellular movement, cancer, connective tissue disorders'. Each interaction is supported by at least one literature reference, with solid lines representing direct interactions and dashed lines representing indirect interactions.
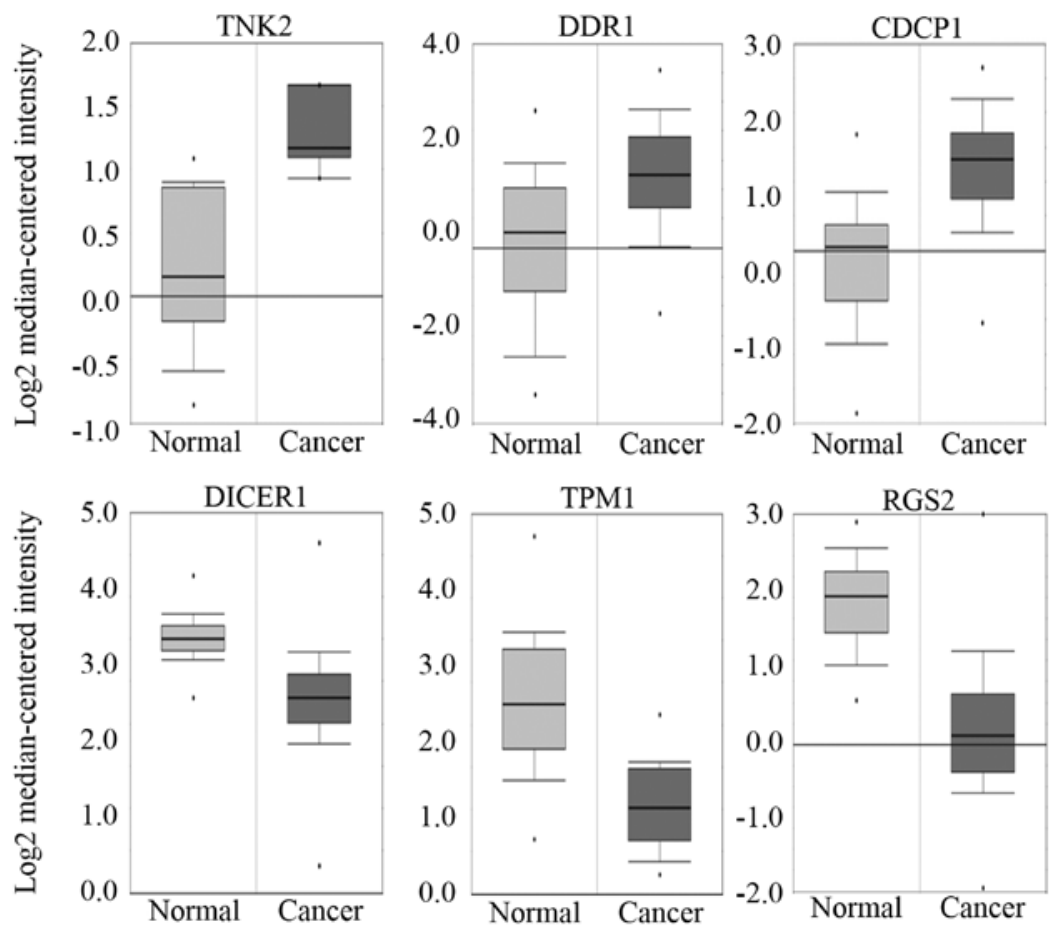

Figure 3. In silico expression profiles of selected genes with altered expression by downregulation of SCTR in breast cancer. Six genes that have shown expression change by downregulation of SCTR using siRNA were selected, and their expression was examined in silico in the expression dataset and presented as a boxplot. A coincidence appeared for the expression between the database and siRNA-treated cells. 
Table II. Genes on top network displaying differential expression by knockdown of SCTR in MCF-10A cells.

\begin{tabular}{|c|c|c|c|}
\hline Gene & Accession no. & Description & Fold change \\
\hline IL8 & NM_000584.2 & Interleukin 8 & 21.17 \\
\hline LAMC2 & NM_005562.1 & Laminin, $\gamma 2$ & 7.17 \\
\hline SDC1 & NM_001006946 & Syndecan 1 & 6.62 \\
\hline ЕРНА2 & NM_004431.2 & EPH receptor A2 & 4.69 \\
\hline LAMB3 & NM_000228.2 & Laminin, $\beta 3$ & 4.64 \\
\hline ABCB6 & NM_005689.1 & ATP-binding cassette, sub-family B & 4.62 \\
\hline ZMAT3 & NM_152240.1 & Zinc finger, matrin-type 3 & 3.83 \\
\hline ANGPTL4 & NM_139314.1 & Angiopoietin-like 4 & 3.38 \\
\hline LAMA3 & NM_198129.1 & Laminin, $\alpha 3$ & 3.31 \\
\hline DDR1 & NM_013993.2 & Discoidin domain receptor tyrosine kinase 1 & 3.30 \\
\hline GALNT3 & NM_004482.2 & GalNAc-T3 & 3.24 \\
\hline SORL1 & NM_003105.3 & Sortilin-related receptor & 3.18 \\
\hline TNK2 & NM_005781.4 & Tyrosine kinase, non-receptor, 2 & 3.16 \\
\hline CDCP1 & NM_022842.3 & CUB domain containing protein 1 & 2.97 \\
\hline PALLD & NM_016081.3 & Palladin, cytoskeletal associated protein & 2.93 \\
\hline $\mathrm{ADM}$ & NM_001124.1 & Adrenomedullin & 2.81 \\
\hline TNC & NM_002160.1 & Tenascin C & 2.76 \\
\hline ABCG1 & NM_016818.2 & ATP-binding cassette, sub-family G (WHITE), member 1 & 2.63 \\
\hline IQGAP1 & NM_003870.3 & IQ motif containing GTPase activating protein 1 & 2.63 \\
\hline LAMA5 & NM_005560.3 & Laminin, $\alpha 5$ & 2.63 \\
\hline TNFRSF12A & NM_016639.1 & Tumor necrosis factor receptor superfamily, member $12 \mathrm{~A}$ & 2.54 \\
\hline LILRB1 & NM_001081637.1 & Leukocyte immunoglobulin-like receptor, subfamily B & 2.51 \\
\hline DICER1 & NM_030621.2 & Dicer 1 , ribonuclease type III & -2.77 \\
\hline PRUNE & NM_021222.1 & Prune homolog (Drosophila) & -3.16 \\
\hline TPM1 & NM_001018020.1 & Tropomyosin 1 & -3.52 \\
\hline RGCC & NM_014059.2 & Regulator of cell cycle & -3.54 \\
\hline RGS2 & NM_002923.1 & Regulator of G-protein signaling 2 & -7.41 \\
\hline
\end{tabular}
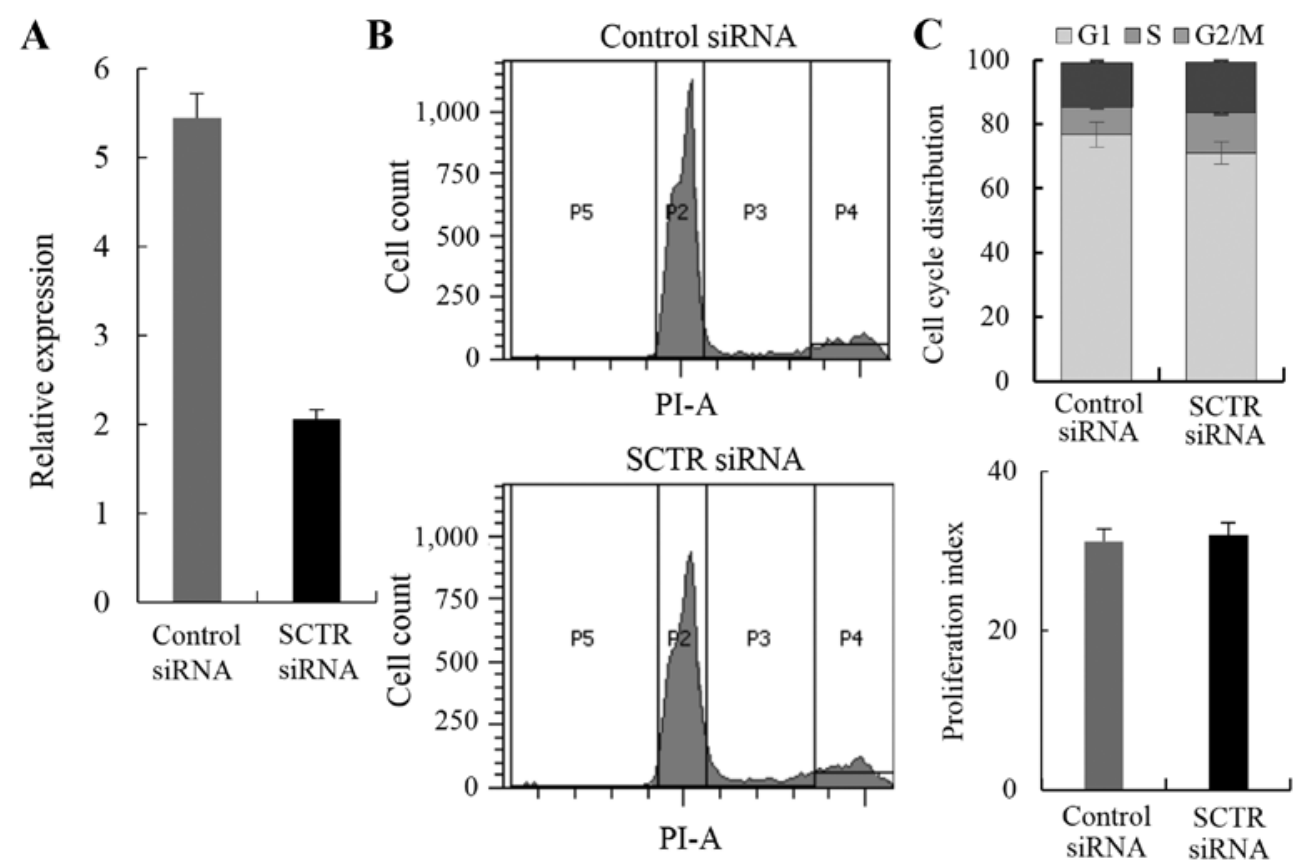

Figure 4. FACS analysis of SCTR-downregulated MCF-10A cells. SCTR was downregulated using siRNA in the normal breast cell line MCF-10A (A) and analyzed for cell proliferation by FACS (B). (C) Cell cycle distribution of cells (top) and proliferation index (bottom) deduced from the FACS result. Control siRNA is the result from transfection of a negative siRNA. 
A
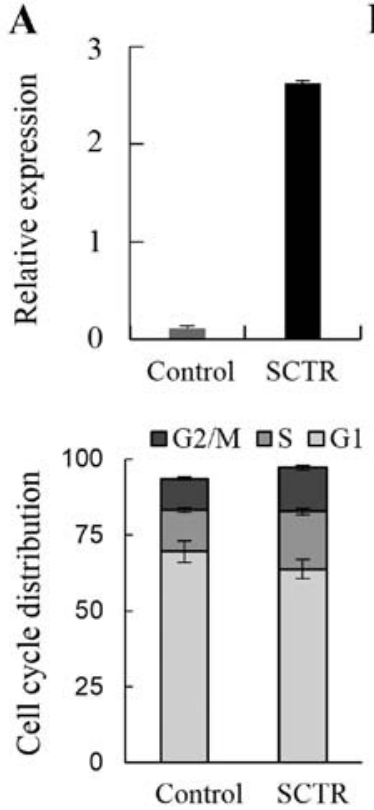

B
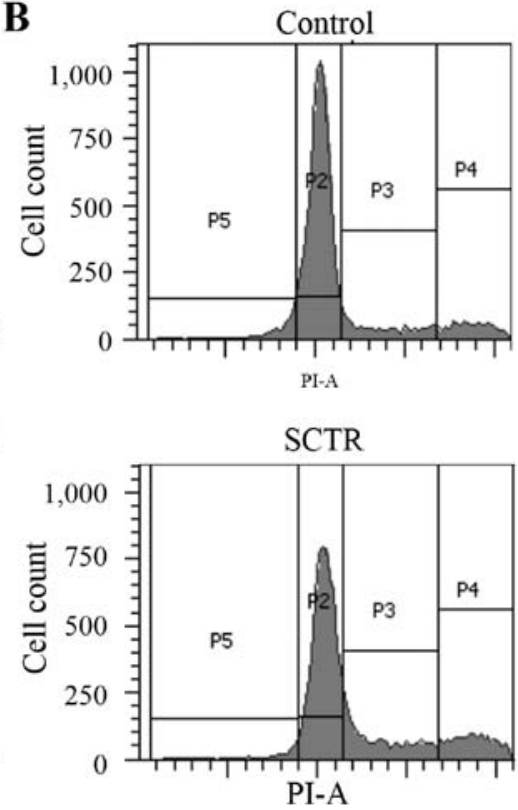

C
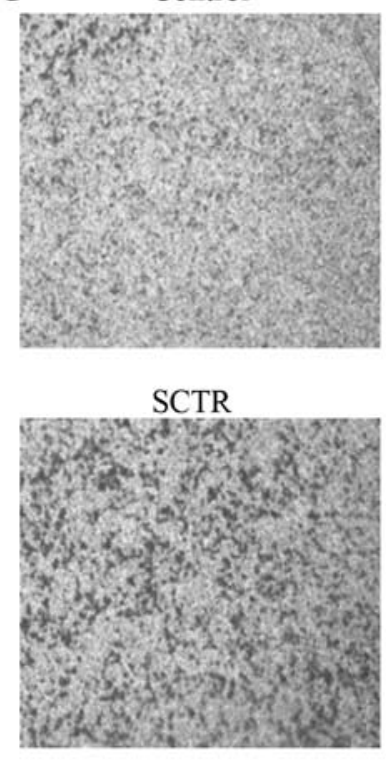

Figure 5. SCTR stimulates cell proliferation and migration of the MCF-7 breast cells. To examine the effect of SCTR on cell proliferation, the gene was overexpressed in the MCF-7, and the cells were analyzed for cell cycle by FACS and for cell migration. (A) RT-PCR analysis of SCTR in MCF-7 cells stably transfected with an SCTR-expressing plasmid (SCTR). Negative control is the result from transfection of the vector alone. (B) FACS analysis of the MCF-7 cells harboring SCTR-expressing plasmid or vector alone. The bar graph at the bottom of panel A shows the ratio of cells at different cell cycle stages. (C) Representative data of cell migration assay. (Original magnification, $\mathrm{x} 40$ ).

stimulates cell migration as well as cell proliferation of the MCF-7 cancer cells by regulating cell cycle-related genes.

\section{Discussion}

GPCRs modulate diverse cellular responses to the majority of neurotransmitters and hormones within the human body (27). Various GPCRs have been found to be overexpressed in primary and metastatic tumors $(28,29)$. In breast cancer, specific GPCR systems are excessively activated in malignant breast cancer due to the overexpression of receptors, which contributes to the progression and spread of breast cancer $(30,31)$. For example, GPR30, a seven membranespanning estrogen receptor, is linked to estrogen binding and heparin-bound epidermal growth factor release, and it induces the proliferation and migration of breast cancer cells through connective tissue growth factor $(32,33)$. For these reasons, GPCRs have long been the most promising targets for developing therapeutic agents (34). For instance, an adhesion GPCR, CD97, was targeted by lysophosphatidic acid in the MDA-MB-231 breast cancer cells (35).

In the present study, many GPCRs showed aberrant methylation, with some being hypermethylated and others being hypomethylated in breast cancer. These differential methylation profiles of the GPCRs should direct their expression in such a way as to specifically contribute to carcinogenesis. In the case of SCTR, a high frequency of methylation at the gene's $\mathrm{CpG}$ island was revealed in colorectal cancer, although its relationship to expression has yet to be determined (36). The roles of SCTR have been elucidated only in a few cancer types. When transfected into Y1 adrenocortical carcinoma cells, overexpressing SCTR stimulated cell proliferation via the PI3K/AKT signaling cascade (37).
Whether SCTR is an oncogene or a tumor suppressor is still controversial. Several studies support the idea that it is an oncogene, as high expression was observed in a few cancer types, such as pancreatic ductal adenocarcinoma and gastrinoma $(13,15)$, whereas, other studies indicate the downregulation of the gene in colorectal cancer, cholangiocarcinoma and prostate cancer $(14,16,17)$. Notably, the differential regulation occurs in a tissue-specific manner, and this drove us to profile the expression in breast cancer.

In the present study, the authors suggested unique regulatory activities of SCTR in breast cells (i.e., tumor-suppressive activity in normal cells and proliferation- and migrationstimulating activity in cancer cells). Several experimental results support our hypothesis. First, the downregulation of SCTR by siRNA in normal breast MCF-10A cells, dysregulated oncogenes or tumor suppressors toward suppressing tumor development; oncogenes were upregulated, and tumor suppressors were downregulated by siRNA. Second, the overexpression of SCTR in MCF-7 breast cancer cells increased the proliferation rate of the cells, while little effect on normal cells was observed when the gene was downregulated. Third, the overexpression of SCTR in MCF-7 cancer cells increased cell migration. The so-called double-edge sword activity of tumor-related genes (i.e., upholding both oncogenic and tumor-suppressive activity) are known for genes involved in key signaling pathways, such as TGF- $\beta 1$ and JNK. TGF- $\beta 1$ is a potent growth inhibitor, with tumor-suppressing activity. Cancers are often refractile to this growth inhibition because of the downstream perturbation of the signaling pathway (38). JNK has pro- or anti-apoptotic functions depending on the cell type, nature of the death stimulus, duration of its activation, and activity of other signaling pathways (39). The molecular mechanism of SCTR for negative and positive regulation in 
terms of the cell proliferation that appeared in normal and cancer cells is to be elucidated in further studies.

The signaling pathway via GPCR is a highly complicated process. NF- $\kappa \mathrm{B}, \beta$-catenin and $\mathrm{PI} 3 \mathrm{~K} \gamma$ are major signaling genes aberrantly regulated in cancer (40). In the present study, IL-8 was upregulated after inhibiting SCTR, implying that SCTR is able to suppress IL- 8 expression. IL-8 is reported to promote breast cancer by increasing cell invasion, angiogenesis, and metastases $(41,42)$. The signaling pathway connecting SCTR and IL-8 should be elucidated in future studies. Genes known to interact with IL-8 in the network (Fig. 2) (e.g., DDR1, TNFRSF12A and DICER1) could be informative. Taken together, it is speculated that SCTR acts as a tumor suppressor in normal breast tissue. However, the gene has a tendency to proliferate breast cancer cells although it is downregulated in cancer by promoter methylation.

\section{Acknowledgements}

The present study was supported by the Basic Science Research Program through the National Research Foundation of Korea (NRF) funded by the Ministry of Education, Science, and Technology (NRF-2012R1A1A2040830). Dr H.S. Kang was supported by a grant provided by the National Cancer Center, Korea.

\section{References}

1. Pierce KL,Premont RT and Lefkowitz RJ: Seven-transmembrane receptors. Nat Rev Mol Cell Biol 3: 639-650, 2002.

2. Reiter E, Ahn S, Shukla AK and Lefkowitz RJ: Molecular mechanism of $\beta$-arrestin-biased agonism at seven-transmembrane receptors. Annu Rev Pharmacol Toxicol 52: 179-197, 2012.

3. Rajagopal S, Ahn S, Rominger DH, Gowen-MacDonald W, Lam CM, Dewire SM, Violin JD and Lefkowitz RJ: Quantifying ligand bias at seven-transmembrane receptors. Mol Pharmacol 80: 367-377, 2011.

4. Urban JD, Clarke WP, von Zastrow M, Nichols DE, Kobilka B, Weinstein H, Javitch JA, Roth BL, Christopoulos A, Sexton PM, et al: Functional selectivity and classical concepts of quantitative pharmacology. J Pharmacol Exp Ther 320: 1-13, 2007.

5. Chevalier N, Bouskine A and Fenichel P: Role of GPER/GPR30 in tumoral testicular germ cells proliferation. Cancer Biol Ther 12: 2-3, 2011

6. Rago V, Romeo F, Giordano F, Maggiolini M and Carpino A: Identification of the estrogen receptor GPER in neoplastic and non-neoplastic human testes. Reprod Biol Endocrinol 9: 135, 2011.

7. Friedl $\mathrm{P}$ and Wolf $\mathrm{K}$ : Tumour-cell invasion and migration: Diversity and escape mechanisms. Nat Rev Cancer 3: 362-374, 2003.

8. Mitra D,Luo X, Morgan A, Wang J, Hoang MP,Lo J, Guerrero CR, Lennerz JK, Mihm MC, Wargo JA, et al: An ultraviolet-radiationindependent pathway to melanoma carcinogenesis in the red hair/ fair skin background. Nature 491: 449-453, 2012.

9. Green JA, Suzuki K, Cho B, Willison LD, Palmer D, Allen CD, Schmidt TH, Xu Y, Proia RL, Coughlin SR, et al: The sphingosine 1-phosphate receptor S1P2 maintains the homeostasis of germinal center B cells and promotes niche confinement. Nat Immunol 12: 672-680, 2011.

10. Chey WY and Chang TM: Secretin, 100 years later. J Gastroenterol 38: 1025-1035, 2003.

11. Chu JY, Lee LT, Lai CH, Vaudry H, Chan YS, Yung WH and Chow BK: Secretin as a neurohypophysial factor regulating body water homeostasis. Proc Natl Acad Sci USA 106: 15961-15966, 2009.

12. Davis RJ,Page KJ, Dos Santos Cruz GJ,Harmer DW, Munday PW, Williams SJ, Picot J, Evans TJ, Sheldrick RL, Coleman RA, et al: Expression and functions of the duodenal peptide secretin and its receptor in human lung. Am J Respir Cell Mol Biol 31: 302-308, 2004.
13. Körner M, Hayes GM, Rehmann R, Zimmermann A, Friess H, Miller LJ and Reubi JC: Secretin receptors in normal and diseased human pancreas: Marked reduction of receptor binding in ductal neoplasia. Am J Pathol 167: 959-968, 2005.

14. Onori P, Wise C, Gaudio E, Franchitto A, Francis H, Carpino G, Lee V, Lam I, Miller T, Dostal DE, et al: Secretin inhibits cholangiocarcinoma growth via dysregulation of the cAMP-dependent signaling mechanisms of secretin receptor. Int J Cancer 127: 43-54, 2010.

15. Ding WQ, Kuntz S, Böhmig M, Wiedenmann B and Miller LJ: Dominant negative action of an abnormal secretin receptor arising from mRNA missplicing in a gastrinoma. Gastroenterology 122: 500-511, 2002.

16. Mayor R, Casadomé L, Azuara D, Moreno V, Clark SJ, Capellà G and Peinado MA: Long-range epigenetic silencing at 2q14.2 affects most human colorectal cancers and may have application as a non-invasive biomarker of disease. Br J Cancer 100: 1534-1539, 2009.

17. Devaney J, Stirzaker C, Qu W, Song JZ, Statham AL, Patterson KI, Horvath LG, Tabor B, Coolen MW, Hulf T, et al: Epigenetic deregulation across chromosome 2 q14.2 differentiates normal from prostate cancer and provides a regional panel of novel DNA methylation cancer biomarkers. Cancer Epidemiol Biomarkers Prev 20: 148-159, 2011.

18. Stefansson OA, Jonasson JG, Olafsdottir K, Hilmarsdottir H, Olafsdottir G, Esteller M, Johannsson OT and Eyfjord JE: CpG island hypermethylation of BRCA1 and loss of pRb as co-occurring events in basal/triple-negative breast cancer. Epigenetics 6: 638-649, 2011.

19. Hino R, Uozaki H, Murakami N, Ushiku T, Shinozaki A, Ishikawa S, Morikawa T, Nakaya T, Sakatani T, Takada K, et al: Activation of DNA methyltransferase 1 by EBV latent membrane protein $2 \mathrm{~A}$ leads to promoter hypermethylation of PTEN gene in gastric carcinoma. Cancer Res 69: 2766-2774, 2009.

20. Sebova K, Zmetakova I, Bella V, Kajo K, Stankovicova I, Kajabova V, Krivulcik T, Lasabova Z, Tomka M, Galbavy S, et al: RASSF1A and CDH1 hypermethylation as potential epimarkers in breast cancer. Cancer Biomark 10: 13-26, 2011-2012.

21. Mahajan K, Coppola D, Chen YA, Zhu W, Lawrence HR, Lawrence NJ and Mahajan NP: Ack1 tyrosine kinase activation correlates with pancreatic cancer progression. Am J Pathol 180: 1386-1393, 2012.

22. Quan J, Yahata T, Adachi S, Yoshihara K and Tanaka K: Identification of receptor tyrosine kinase, discoidin domain receptor 1 (DDR1), as a potential biomarker for serous ovarian cancer. Int J Mol Sci 12: 971-982, 2011.

23. Miyazawa Y, Uekita T, Ito Y, Seiki M, Yamaguchi H and Sakai R: CDCP1 regulates the function of MT1-MMP and invadopodiamediated invasion of cancer cells. Mol Cancer Res 11: 628-637, 2013.

24. Díaz-García CV, Agudo-López A, Pérez C, López-Martín JA, Rodríguez-Peralto JL, de Castro J, Cortijo A, MartínezVillanueva M, Iglesias L, García-Carbonero R, et al: DICER1, DROSHA and miRNAs in patients with non-small cell lung cancer: Implications for outcomes and histologic classification. Carcinogenesis 34: 1031-1038, 2013.

25. Bharadwaj S and Prasad GL: Tropomyosin-1, a novel suppressor of cellular transformation is downregulated by promoter methylation in cancer cells. Cancer Lett 183: 205-213, 2002.

26. Wolff DW, Xie Y, Deng C, Gatalica Z, Yang M, Wang B, Wang J, Lin MF, Abel PW and Tu Y: Epigenetic repression of regulator of G-protein signaling 2 promotes androgen-independent prostate cancer cell growth. Int J Cancer 130: 1521-1531, 2012.

27. Rosenbaum DM, Rasmussen SG and Kobilka BK: The structure and function of G-protein-coupled receptors. Nature 459: 356-363, 2009.

28. O'Hayre M, Degese MS and Gutkind JS: Novel insights into G protein and $\mathrm{G}$ protein-coupled receptor signaling in cancer. Curr Opin Cell Biol 27: 126-135, 2014.

29. Lappano R and Maggiolini M: G protein-coupled receptors: Novel targets for drug discovery in cancer. Nat Rev Drug Discov 10: 47-60, 2011.

30. Müller A, Homey B, Soto H, Ge N, Catron D, Buchanan ME, McClanahan $\mathrm{T}$, Murphy $\mathrm{E}$, Yuan $\mathrm{W}$, Wagner SN, et al: Involvement of chemokine receptors in breast cancer metastasis. Nature 410: 50-56, 2001.

31. Wülfing P, Kersting C, Tio J, Fischer RJ, Wülfing C, Poremba C, Diallo R, Böcker W and Kiesel L: Endothelin-1-, endothelin-A-, and endothelin-B-receptor expression is correlated with vascular endothelial growth factor expression and angiogenesis in breast cancer. Clin Cancer Res 10: 2393-2400, 2004. 
32. Filardo EJ, Graeber CT, Quinn JA, Resnick MB, Giri D, DeLellis RA, Steinhoff MM and Sabo E: Distribution of GPR30, a seven membrane-spanning estrogen receptor, in primary breast cancer and its association with clinicopathologic determinants of tumor progression. Clin Cancer Res 12: 6359-6366, 2006.

33. Pandey DP, Lappano R, Albanito L, Madeo A, Maggiolini M and Picard D: Estrogenic GPR30 signalling induces proliferation and migration of breast cancer cells through CTGF. EMBO J 28 : 523-532, 2009

34. Lagerström MC and Schiöth HB: Structural diversity of G protein-coupled receptors and significance for drug discovery. Nat Rev Drug Discov 7: 339-357, 2008.

35. Park SJ, Lee KP, Kang S, Chung HY, Bae YS, Okajima F and Im DS: Lysophosphatidylethanolamine utilizes LPA(1) and CD97 in MDA-MB-231 breast cancer cells. Cell Signal 25: 2147-2154, 2013.

36. Karpinski P, Ramsey D, Grzebieniak Z, Sasiadek MM and Blin N: The $\mathrm{CpG}$ island methylator phenotype correlates with long-range epigenetic silencing in colorectal cancer. Mol Cancer Res 6: 585-591, 2008
37. Lee M, Waser B, Reubi JC and Pellegata NS: Secretin receptor promotes the proliferation of endocrine tumor cells via the PI3K/ AKT pathway. Mol Endocrinol 26: 1394-1405, 2012.

38. Oft M, Peli J, Rudaz C, Schwarz H, Beug H and Reichmann E: TGF-betal and Ha-Ras collaborate in modulating the phenotypic plasticity and invasiveness of epithelial tumor cells. Genes Dev 10: 2462-2477, 1996

39. Liu J and Lin A: Role of JNK activation in apoptosis: A doubleedged sword. Cell Res 15: 36-42, 2005.

40. Wu J, Xie N, Zhao X, Nice EC and Huang C: Dissection of aberrant GPCR signaling in tumorigenesis - a systems biology approach. Cancer Genomics Proteomics 9: 37-50, 2012

41. Chen Y, Chen L, Li JY, Mukaida N, Wang Q, Yang C, Yin WJ, Zeng $\mathrm{XH}$, Jin $\mathrm{W}$ and Shao ZM: ER $\beta$ and PEA3 co-activate IL-8 expression and promote the invasion of breast cancer cells. Cancer Biol Ther 11: 497-511, 2011.

42. Kim H, Choi JA, Park GS and Kim JH: BLT2 up-regulates interleukin-8 production and promotes the invasiveness of breast cancer cells. PLoS One 7: e49186, 2012. 